\title{
1 On the temporally flexible structure of plant-pollinator interaction networks
}

3 Paul J. CaraDonna ${ }^{1,2,3}$ \& Nickolas M. Waser ${ }^{1,4}$

4

$5 \quad{ }^{1}$ Rocky Mountain Biological Laboratory, Crested Butte, CO, USA

$6 \quad{ }^{2}$ Chicago Botanic Garden, Glencoe, IL, USA

$7 \quad$ 3Plant Biology and Conservation, Northwestern University, Evanston, IL, USA

$8{ }^{4}$ School of Natural Resources and the Environment, University of Arizona, Tucson, AZ, USA

10 Author for correspondence email:

11 pcaradonna@chicagobotanic.org

12 
14 Abstract. Ecological communities consist of species that are joined in complex networks of

15 interspecific interaction. The interactions that networks depict often form and dissolve rapidly,

16 but this temporal variation is not well integrated into our understanding of the causes and

17 consequences of network structure. If interspecific interactions exhibit temporal flexibility across

18 time periods over which organisms co-occur, then the emergent structure of the corresponding

19 network may also be temporally flexible, something that a temporally-static perspective would

20 miss. Here, we use an empirical system to examine short-term flexibility in network structure

21 (connectance, nestedness, and specialization), and in individual species interactions that

22 contribute to that structure. We investigated weekly plant-pollinator networks in a subalpine

23 ecosystem across three summer growing seasons. To link the interactions of individual species to

24 properties of their networks, we examined weekly temporal variation in species' contributions to

25 network structure. As a test of the potential robustness of networks to perturbation, we also

26 simulated the random loss of species from weekly networks. We then compared the properties of

27 weekly networks to the properties of cumulative networks that aggregate field observations over

28 each full season. A week-to-week view reveals considerable flexibility in the interactions of

29 individual species and their contributions to network structure. For example, species that would

30 be considered relatively generalized across their entire activity period may be much more

31 specialized at certain times, and at no point as generalized as the cumulative network may

32 suggest. Furthermore, a week-to-week view reveals corresponding temporal flexibility in network

33 structure and potential robustness throughout each summer growing season. We conclude that

34 short-term flexibility in species interactions leads to short-term variation in network properties,

35 and that a season-long, cumulative perspective may miss important aspects of the way in which

36 species interact, with implications for understanding their ecology, evolution, and conservation. 


\section{KEYWORDS}

connectance, interaction turnover, nestedness, robustness, specialization, temporal ecology, seasonality, subalpine

\section{INTRODUCTION}

Ecological communities are characterized not only by the identities and relative

abundances of their component species, but also by the interactions among these species. These interspecific interactions often change perceptibly through time, as individual organisms transition through stages of their life histories and as seasons progress. As Charles Elton (1927,

p. 96) observed, "Since the biological environment is constantly shifting with the passage of the seasons, it follows that the food habits of animals often change accordingly". interaction network, whose topological structure may then be related to patterns of biodiversity, evolution of component species, and community function (e.g., Bascompte et al. 2006; Bastolla et al. 2009; Thébault \& Fontaine, 2010; Rohr et al. 2014; Schleuning et al. 2015). To date, the most common perspective on ecological networks is one that in effect assigns fixed values to interactions and to aspects of structure over relatively long time scales (e.g., entire growing seasons: Bascompte \& Stouffer, 2009; Blonder et al. 2012; Burkle \& Alarcón, 2011; Poisot et al. 2015; McMeans et al. 2015; Trøjelsgaard \& Olesen, 2016). Such a temporally-static perspective offers many insights into ecological communities. However, it also stands to overlook more rapid

58 time scales at which interactions form and dissolve in many systems, as individual organisms (or

59 their life stages that interact) appear and disappear, and as interactions among those present at the 60 same time shift and rewire (sensu Elton, 1927; McMeans et al. 2015; CaraDonna et al. 2017). If 61 interspecific interactions exhibit temporal flexibility across time periods over which organisms 
co-occur, then the emergent structure of the corresponding networks may also be temporally flexible, something that a temporally-static perspective is likely to miss.

Temporal variation in the structure of interaction networks has indeed been documented at multiple scales, from days, to weeks, to months, years, and beyond (Winemiller, 1990; Alárcon et al. 2006; Olesen et al. 2008; Burkle \& Alarcón, 2011; Trøjelsgaard \& Olesen, 2016). The few studies that have examined the consequences of short-term temporal variation in network structure suggest that it is an important component of community dynamics and species coexistence (Saavedra et al. 2016a; Saavedra et al. 2016b; Saavedra et al. 2017). Yet, overall, it still remains unclear what information is gained from different temporal perspectives, and whether the structural properties of networks are temporally consistent (Trøjelsgaard \& Olesen, 2016).

Here, we explore the magnitude and consistency of week-to-week variation in plantpollinator networks in a subalpine ecosystem. Because species and their interactions turn over rapidly in this system (CaraDonna et al. 2017), we focus on short time periods when species are known to co-occur and have the opportunity to interact. To better link the actions of individual organisms to the emergent structural properties of their networks, we also investigate temporal variation in aspects of the interactions of individual species and their contribution to overall network structure in each week. To these ends, we use nearly 29,000 observations of pollinators visiting flowers recorded across three successive summer growing seasons. These observations yield 42 pollination networks resolved at the scale of single weeks, for each of which we quantified aspects of network structure and species' roles within these networks. With these data we (1) quantify temporal variation in network properties from week to week across the growing season; (2) explore how network properties calculated at finer, biologically-relevant time scales compare to those calculated from cumulative, season-long networks; and (3) ask whether weekly 
networks exhibit temporal variation in their apparent robustness to the simulated loss of species.

Our analyses shed light on some aspects of temporal consistency — and inconsistency — of network properties, and suggest connections between the interactions of individual species and the emergent structural properties of networks containing those species.

\section{MATERIALS AND METHODS}

Study site and system. We worked at The Rocky Mountain Biological Laboratory (RMBL) in Gothic, Colorado, USA $\left(38^{\circ} 57.5^{\prime} \mathrm{N}, 106^{\circ} 59.3^{\prime} \mathrm{W}, 2900 \mathrm{~m}\right.$ a.s.1.) over the summers of 2013,2014 , and 2015. The RMBL is a mosaic of wet and dry subalpine meadows intermixed with aspen and conifer forest. This subalpine system is exemplified by temporal change (CaraDonna et al. 2017). It is highly seasonal, with long winters devoid of most biological activity and a short summer growing season of 3-5 months (CaraDonna et al. 2014). Within the summers a series of plant species flower in succession, and insect and hummingbird pollinators enter the system via eclosion and immigration and leave it via death, diapause, and emigration.

\section{Plant-pollinator observations. CaraDonna et al. (2017) provide a detailed description of the} sampling protocol and its justification. In brief, we observed interactions between flowers and insect and hummingbird pollinators at weekly intervals for the majority of all of the three summers ( $n=12$ weeks in 2013, 15 in 2014, 15 in 2015). All observations took place in two adjacent dry meadows that cover ca. 2800 and $3015 \mathrm{~m}^{2}$ and are separated by ca. $100 \mathrm{~m}$ of aspen

forest. Observations began about one week after snowmelt in each summer, coinciding with the first emergence of flowers and pollinators. Within each week, we conducted 32 15-min observations for a total of 8 hours per week; interaction rarefaction curves and abundance-based richness estimators indicated that this sampling effort sufficed to detect most (on average 85- 
$11093 \%$ ) of the pairwise interactions that occurred in each week (see CaraDonna et al. 2017 for

111 details). Each complete weekly census took place over 2-3 consecutive days and was separated

112 from the start of the next census by 3-5 days. We randomly selected one of four quadrants within

113 each meadow during each 15-min observation period, then sampled the remaining quadrants in

114 random order, and then repeated this in the other meadow. During each 15-min period we walked

115 around the focal quadrant and recorded all observed plant-pollinator interactions. We defined an

116 interaction as a visitor of any species unambiguously contacting the reproductive structures of a

117 flower; we refer to floral visitors as pollinators while recognizing that their quality as mutualists

118 may vary widely. A single weekly plant-pollinator interaction network was constructed from the

119 observations of each week. A cumulative plant-pollinator network was constructed by

120 aggregating all observations across each entire summer growing season. All flowering plants

121 were identified to species, and all pollinators to species or to the finest taxonomic level possible

122 under field conditions (for simplicity, we refer to all taxonomic levels as "species" in what

123 follows). Pollinators were not collected during observations to prevent artefacts of destructive

124 sampling.

126 Structure of pollination networks. For all 42 weekly networks and for all three cumulative,

127 season-long networks, we investigated temporal variation in three metrics that describe different

128 aspects of the structure of interactions: (i) connectance; (ii) network-level specialization; and (iii)

129 network nestedness. For network connectance and nestedness, we calculated both binary

130 (unweighted) and frequency-based (weighted) metrics (network specialization is already a

131 frequency-based metric); because overall patterns were qualitatively similar when we calculated

132 either binary or frequency-based metrics for connectance and nestedness, we limit description and 
133 discussion of frequency-based metrics in the main text for brevity (details and values for

134 weighted metrics are given in Appendix S1: Table S1).

Connectance describes a basic component of network complexity and is calculated as the

136 proportion of observed links out of all possible links in the network (values range from 0 to 1 ).

137 Observed connectance values are frequently correlated with network size (i.e., the number of

138 species in the network), so we additionally calculated an estimate of connectance adjusted for

139 size. To do this, we extracted the residuals from a regression of network size by connectance,

140 which effectively removes any variation due to size. Values greater or less than zero respectively

141 indicate that the network is more or less connected than expected after accounting for its size.

142 Network-level specialization, $\mathrm{H}_{2}$, is a frequency-based metric that characterizes the level

143 of interaction specialization within a bipartite network (Blüthgen et al. 2006). If we consider

144 interactions as a feature of the ecological niche, network-level specialization describes the extent

145 of niche partitioning across plants and pollinators in their use of mutualistic partners. Values of

$146 H_{2}$ ' are based upon the degree to which the observed interactions in the network deviate from

147 those that would be expected if they occurred at random (holding marginal sums constant).

148 Values range from 0 to 1 with higher values indicating greater specialization, and therefore less

149 niche overlap. $\mathrm{H}_{2}$ ' effectively accounts for sources of variation related to network size and

150 connectance, and can be considered a scale-independent metric that characterizes network

151 specialization; it is appropriate for across-network comparison (Blüthgen et al. 2006).

Network nestedness describes the extent to which specialization species (those with few

153 links) interact with subsets of the species that generalists (those with many links) interact with

154 (Bascompte, Jordano, Melián, \& Olesen, 2003). We calculated nestedness following the NODF

155 (nestedness metric based on overlap and decreasing fill) algorithm of Almeida-Neto, Guimarães,

156 Guimarães, Loyola, and Ulrich (2008). Values range from 0 to 100, where 100 theoretically 
157 indicates a perfectly nested network. However, the actual maximum nestedness of a given

158 network is often less than this theoretical maximum, which complicates the comparison of values

159 across networks (Song, Rohr, \& Saavedra, 2017). Furthermore, nestedness can be influenced by

160 the size of the network and its connectance (Song et al. 2017). To account for these three issues

161 we followed the methods of Song, Rohr, and Saavedra $(2017,2019)$ and Simmons, Hoeppke, and

162 Sutherland (2019) to calculate the combined nestedness value. This metric provides an estimate

163 of nestedness that can be appropriately compared across networks of different sizes and observed

164 connectance.

165 In addition to examining week-to-week variation, we compared the mean value of each

166 network metric averaged across all weeks in each summer growing season to the value from the

167 single cumulative network for that summer, using one-sample t-tests. For both network

168 specialization $\left(\mathrm{H}_{2}{ }^{\prime}\right)$ and nestedness $(\mathrm{NODF})$, we examined the extent to which the observed

169 values deviated from those generated at random using a null model with the following

170 constraints: (i) the number of links within each network (i.e., observed connectance) was held

171 constant, and (ii) links were then randomised under the constraint that interactions for each pair of

172 plant $i$ and pollinator $j$ occurred in proportion to the product of their interaction degrees (for

173 binary webs; Bascompte et al. 2003), or in proportion to the frequency of visits between them (for

174 frequency-based webs; Vázquez et al. 2007). We compared observed values to those generated

175 from 100 simulated interaction matrices.

177 Species contributions to network structure. We explored temporal variation in three measures of

178 how interactions of individual species contribute to structure of their networks: (i) the number of

179 links per species; (ii) species-level interaction specialization; and (iii) species nestedness

180 contribution. We included only species that were present over two or more weeks in at least one 
181 of three summers. Links per species (i.e., species interaction degree) is simply the number of

182 mutualistic partners a species interacts with. Because the number of links may be influenced by

183 the number of available partners (i.e., resources), we additionally calculated normalized degree,

184 which is the proportion of realised links for a given species. Species interaction specialization, $d^{\prime}$,

185 is a measure of interaction niche breadth of a given species (Blüthgen et al. 2006). As with

186 network-level specialization $\left(H_{2}{ }^{\prime}\right), d^{\prime}$ is based upon how strongly the interactions of a species

187 deviate from those occurring at random among its available mutualistic partners, and accounts for

188 sources of variation related to network size. Values range from 0 to 1 with higher values

189 indicating greater specialization, and therefore narrower interaction niche breadth and less niche

190 overlap between species. The relative contribution of a given species to overall nestedness

$191(N O D F)$ is calculated as the extent to which the nested structure of the network changes with the

192 randomization of the interactions of the focal species (following Saavedra et al. 2011). Individual

193 values are z-scores, which can range from positive to negative, indicating that a species has a

194 positive or negative effect, respectively, on the network nestedness.

196 Network robustness to simulated species extinction. As a heuristic approach to explore potential

197 network robustness to cascading extinctions, we simulated the random loss of species from both

198 weekly and season-long networks. We use the term "potential" here in recognition that secondary

199 extinctions are likely to play out more slowly than week-to-week. The simulations tabulated

200 secondary species extinctions as a consequence of the sequential loss of (i) plant species, (ii)

201 pollinators, or (iii) both plants and pollinators simultaneously. Simulations removed species from

202 networks in random order; when remaining species lost all interaction partners they were counted

203 as secondary extinctions. We calculated network robustness as the area underneath the extinction

204 curve (Memmott et al. 2004; Burgos et al. 2007); resulting values range from 0 to 1, where 0 
205 indicates that all species become secondarily extinct after the first removal of a species (zero

206 robustness), and 1 indicates that no species become secondarily extinct (complete robustness).

207 Each simulation scenario was run 100 times for each network and robustness values were

208 averaged across these runs. This method for assessing robustness treats the links within each

209 network as unchanging, except when they are lost altogether. Although more complex methods

210 are available, this simple approach serves our purpose, which is to raise the possibility that

211 temporal variation in network structure has consequences that are more far-reaching than is

212 evident week-to-week.

214 Effects of network size and species abundance. Because metrics can be sensitive to network size

215 (Vázquez et al. 2010), we also examined the relationship between each metric from each week

216 and species richness of the network for that week. We also examined the relationship between the

217 estimated abundance of each plant and pollinator species in each week and the metrics that

218 describe its ecological role within the network in that week (CaraDonna et al. 2017 gives details

219 of species-abundance measurements). Relationships were examined with Pearson product

220 moment correlations

221

222 Data analysis. All analyses where conducted in the R statistical computing environment (R Core

223 Team 2018). All network-level and species-level analyses, as well as secondary extinction

224 simulations, were conducted using the R package 'bipartite' (Dormann et al. 2008; Dormann et al.

225 2009; Dormann, 2011).

\section{RESULTS}

228 In total we analyzed 45 interaction networks (42 weekly and 3 cumulative, season-long 
networks), comprising 28,959 individual visitation events representing 547 unique links between

23046 plant and 93 pollinator species. The mean parameter estimates and ranges of values for the

231 weekly networks were largely consistent across all three summers, as were the structural

232 properties of the three cumulative networks (Tables 1; see Appendix S1: Table S2 for additional

233 details on plant, pollinator, and interaction richness for weekly and cumulative networks).

235 Structure of cumulative pollination networks. The 2013, 2014, and 2015 cumulative networks

236 exhibited structural properties similar to those reported for many other mutualistic networks

237 (Jordano, 1987; Vázquez et al. 2009; Thébault \& Fontaine, 2010; Bascompte \& Jordano, 2014;

238 Valdovinos, 2019). Across all three summers, cumulative networks had relatively low values of

239 connectance, a small average number of links per species (2.5 to 4.0), intermediate nested

240 structure, and intermediate network-level specialization (Table 1).

242 Structure of weekly pollination networks. The structural properties of weekly networks were

243 highly variable within each of the three summers (Figs. 1 and 2). This within-season variation

244 was consistent across the summers (Table 1; Appendix S1: Tables S1, S2). For all metrics,

245 weekly values spanned a wide range that was not reflected by the corresponding cumulative

246 estimate (Table 1, Fig. 2). Cumulative connectance was much lower than mean weekly

247 connectance; however, after accounting for network size, this relationship was reversed in that

248 size-adjusted cumulative connectance was generally greater than weekly connectance (Table 1,

249 Fig. 2). Cumulative network-level specialization $\left(\mathrm{H}_{2}{ }^{\prime}\right)$ tended to be similar to mean weekly

250 specialization, with the exception of one summer (2014) in which the cumulative value was

251 lower. Cumulative nestedness $(N O D F)$ tended to be lower than mean weekly nestedness,

252 although this pattern varied from summer to summer, whereas nestedness adjusted for size and 
connectance was more consistently lower for cumulative compared to weekly networks. All

254 patterns were qualitatively similar for frequency-based metrics (Appendix S1: Table S1, Figs.

255 S1-S3).

The observed values of network specialization $\left(H^{2}\right)$ for all three cumulative networks and

257 all but one of the 42 weekly networks were significantly different from the values generated

258 under our null model (Appendix S1: Table S3). Similarly, for nestedness (NODF), all three

259 cumulative networks, and all but one of the 42 weekly networks were significantly nested. For

260 weighted nestedness (weighted $N O D F$ ), all networks were non-nested, a pattern that is consistent

261 with many other mutualistic networks (see Staniczenko et al. 2013; Appendix S1: Table S3).

262

263 Species contributions to cumulative network structure. By being present over two or more weeks

264 in at least one of three summer growing seasons, 72 pollinator species and 33 plant species met

265 our criteria to be included in species-level analyses of their interactions and contributions to

266 network structure. Few plants and pollinators were active for the entire summer growing season

267 (Fig. 1; plant flowering duration: mean across seasons $=3.9$ weeks, range $=1$ to 12 weeks;

268 pollinator activity duration: mean across seasons $=6.01$, range $=1$ to 15 ). The cumulative,

269 season-long number of links (species degree) across plants species varied from 2 to 37 (mean $=$

2709.8 links $)$ and across pollinator species from 2 to $22($ mean $=6.0)$. Cumulative, species-level

271 interaction specialization $\left(d^{\prime}\right)$ was on average moderate (plant mean $=0.43$; pollinator mean $=$

272 0.33), but values ranged from 0.10 to 0.84 across plant species and from zero to 0.85 across

273 pollinator species. The contribution of individual species to the nested structure of cumulative

274 networks was on average positive but ranged across species from positive to negative for both

275 plants $(\mathrm{z}$-score mean $=0.72$; range -4.00 to +4.07$)$ and pollinators $(\mathrm{z}$-score mean $=0.85$; range

$276-2.17$ to +2.72$)$. 
278 Species contributions to weekly network structure. Species-level interaction metrics within

279 weekly networks spanned a wide range of parameter values that were not always similar to values

280 from cumulative networks (Figs. 3, 4; Appendix S1: Figs. S3-S6). Averaging across the three

281 summers, the cumulative number of links per species (i.e., degree) for plants and pollinators

282 tended to be greater than the range of weekly values: for plants, $84 \%$ of the cumulative numbers

283 of links per species were greater than the maximum weekly value, and for pollinators, only $88 \%$

284 of cumulative values were greater than the maximum weekly value (in all cases, the cumulative

285 value was at least as great as the maximum weekly value). However, when accounting for the

286 number of available resources (i.e., normalized degree), for plants, $28.4 \%$ of the cumulative

287 values fell outside the range of the weekly values, and for pollinators, $42.6 \%$ of cumulative values

288 did so. For neither plants nor pollinators was there a clear direction in the difference between

289 cumulative interaction specialization $(d$ ') values and weekly values; for plant interaction

290 specialization $\left(d^{\prime}\right), 39.5 \%$ of cumulative values fell outside the range of weekly values, whereas

291 for pollinators $32.7 \%$ of cumulative values did so. The cumulative nestedness contribution for

292 both individual plant and pollinator species tended to be greater than the mean of their weekly

293 values; for plants, $49.4 \%$ of cumulative values fell outside the range of weekly values, whereas

294 for pollinators only $64.5 \%$ of cumulative values did so.

Network robustness to simulated species extinction. Potential robustness to secondary extinctions

297 for cumulative networks was similar when we removed either plants or pollinators in random

298 order, but was lower when we simultaneously removed both plants and pollinators; these patterns

299 were similar across the three growing seasons (Table 1, Figs. 5; Appendix S1: Figs. S8, S9). Our

300 measure of potential robustness varied across weekly networks, and mean values across weeks 
301 tended to fall below the value for the corresponding cumulative network for all three extinction

302 scenarios (removal of plants, pollinators, or both; Fig. 5).

Effects of network size and species abundance. The relationship between network size and

305 interaction structure depended on the network metric being considered (Appendix S1: Table S1).

306 Connectance in each week was consistently negatively correlated with network size in all three

307 summers. Network-level specialization $\left(\mathrm{H}_{2}{ }^{\prime}\right)$ was not related to network size in any summer (as

308 expected; Blüthgen et al. 2006). Nestedness and network size were negatively correlated,

309 although the strength of this relationship varied across summers. These patterns were qualitatively

310 similar for unweighted and weighted networks (Appendix S1: Table S1). Network size tended to

311 be weakly correlated with network robustness, and the direction of this correlation was

312 inconsistent (Appendix S1: Table S1).

313 The numbers of links per species (species-level degree) tended to be moderately positively

314 correlated with the estimated abundance of each species in each week (mean across species and

315 years, $r=0.55)$. Species-level specialization $\left(d^{\prime}\right)$ was not related to species abundance for most

316 species in any summer $(r=-0.06)$. Species-level nestedness contribution was consistently weakly

317 correlated with species abundance in each week $(r=-0.24)$. All species-specific correlation

318 coefficients for each metric are included in Appendix S1: Table S4.

\section{DISCUSSION}

Our findings highlight considerable fine-scale temporal variability in network structure.

322 The way in which species interact within networks vary substantially from week to week, and this

323 flexibility of interactions, over time periods when species actually co-occur in the subalpine

324 meadows, leads to constant change in network structure throughout the summer growing season. 
325

326

327

328

329

330

In contrast, this biologically-relevant variation is hidden in our cumulative, season-long networks.

Furthermore, cumulative networks may not recover structural patterns that resemble even the averages from networks depicted on finer time scales. For example, weekly networks were consistently more sparsely connected than cumulative networks, after accounting for variation in network size. This pattern reflects the fact that the species themselves have fewer interactions in any given week than they do across their entire activity periods, so that weekly networks are never as richly connected as a cumulative network might suggest. Weekly networks also tended to exhibit overall greater nestedness than cumulative networks, after accounting for network size and connectance. This is consistent with our finding that on average, across both plants and pollinators, individual species' contributions to nestedness tended to be positive from week to week - that is, their interactions with other species fostered a more nested network. Network specialization exhibited the greatest range of week-to-week variation, although for this metric the mean of weekly values tended to more closely resemble cumulative network specialization. On the other hand, the substantial weekly variation in network specialization indicates that the extent to which interaction niches are partitioned can be highly changeable through time.

The temporally-variable structure of weekly networks is ultimately driven by the species that make up these networks and that are constantly modifying their interactions as the season progresses (CaraDonna et al. 2017). To illustrate, Erigeron speciosus (tall fleabane daisy) received visits from individuals of 16 different pollinator species across its 7-week flowering period but in any given week was visited by $1-8$ species, and on average was visited by 5 ; its interaction niche was overall moderately generalized, but ranged from being highly generalized to moderately specialized within any given week (Fig. 3). Similarly, Bombus bifarius (two-form bumble bee) visited 15 different plant species across its entire 15-week activity period but in any given week visited 1-9 species, and on average visited 4; its interaction niche was overall 

examples represent a relatively common plant and a common pollinator in a single summer

351 (2014), but analogous patterns emerge for other plants and pollinators, and in other summers

352 (Figs. 3, 4; Appendix S1: Figs. S3-S6). Thus, species that would be considered relatively

353 generalized in their interactions across their entire activity period may be much more specialized

354 at certain times, and at no point as generalized as the cumulative network may suggest. This

355 species-level temporal variability also translates into how a given species contributes to the nested structure of the network. Overall, many plant and pollinator species contributed positively to network nestedness across their activity periods, but week-to-week contributions for many species ranged from positive to neutral to negative - another difference that derives from within the network that can change rapidly over the course of a season.

362 the species present in a community, and how they interact with others, are both changing through 363 time (Fig. 1; CaraDonna et al. 2017). In many ecosystems species are active only for portions of

364 longer seasons (e.g., Olesen et al. 2008; Petanidou et al. 2008; Carnicer et al. 2009). Within these 365 periods of biological activity, individuals of the species may vary their interactions at fine-time

366 scales in response to changing resource abundances and accessibility as well as the resource use 367 of competing individuals. The pollinators we studied experience rapid changes in the identities 368 (and thereby phenotypes) and abundances of flowers (CaraDonna et al. 2017). In such a system 369 we expect flexibility and opportunism, conditioned by sensory and cognitive abilities or 370 constraints, to dictate who visits whom on scales of seconds to minutes to hours (e.g., Pleasants, 371 1981; Waser et al. 1996; Waser et al. 2018; Chittka \& Thomson, 2001). It is therefore 372 unsurprising that the structural properties of networks fluctuate as the exact mixture of co- 
occurring species shifts, and as species differ in their patterns of interaction within this shifting community context.

when the network is less connected, less nested, and more specialized, its consequences might be

393 reproduction of an individual plant will depend on its immediate pollinator environment;

394 likewise, the fitness of a given pollinator will depend on the floral resources immediately

395 available for consumption. Assuming that a species is rigidly embedded within a temporally

396 aggregated, season-long network overlooks the specific biotic (and abiotic) conditions that should 
397 directly give rise to reproductive success or failure of individuals, ultimately influencing

398 population dynamics and natural selection on traits that mediate interactions. From a conservation

399 perspective, our results suggest that we should carefully consider the temporal variation in how

400 species are linked within a network. For example, even though a species may be relatively

401 generalized across its entire activity period, effective conservation may be contingent upon

402 management at particular times in a season when individuals have limited access to resources and

403 are perhaps most vulnerable. From a multispecies perspective, understanding temporal variation

404 in network structure may similarly highlight parts of the season when numerous species — and

405 therefore the entire community — are collectively most sensitive to disturbance.

Accepting that ecological networks constructed on any temporal scale are ultimately

407 abstractions of an ecological community, is there a timescale that yields the most "true"

408 representation of network structure? We think not, because appropriate temporal resolution will

409 depend on how slowly or quickly systems change and on the questions being asked. As Stigler

410 (2016) explains in tracing the history of aggregation in statistics, aggregation reveals central

411 tendencies and other large patterns in data that otherwise are not apparent. But aggregation can

412 shift the focus away from underlying variation (sensu Iler et al. 2013; Sajjad et al. 2017).

413 Certainly temporal (and spatial) variation in structural properties of interaction networks is not

414 recoverable from single cumulative estimates. We assembled networks across several days within

415 individual weeks, which is biologically reasonable for a subalpine ecosystem in which the season

416 is short, as are the flowering periods of many plant species and the activity periods of many

417 pollinating insects. For other systems, such as those in more slowly-changing tropical climates

418 (e.g., Vizentin-Bugoni et al. 2014; Cuartas-Hernández \& Medel 2015), assembling networks over

419 longer periods such as months may better capture structural properties and species roles. Many

420 ecological interactions other than pollination - for example, interactions between different plant 
421 species (such as competition and ultimate competitive exclusion involving water, light, or

422 nutrients) and between plants and fungi (such as the formation or dissolution of associations

423 between roots and mycorrhizae) — might also proceed at slow rates, so that cumulative networks

424 might best capture them. The most appropriate temporal resolution will also depend on the

425 question being addressed. Temporal aggregation of networks over months or longer may be

426 appropriate for seeing how species or entire systems respond to periods that vary strongly in

427 abiotic and biotic contexts, for example, wet vs. dry seasons, summer vs. winter, or even dramatic

428 land-use change over many years (Winemiller, 1990; Burkle et al. 2013; Cuartas-Hernández \&

429 Medel 2015; Saavedra et al. 2016b; McMeans et al. 2019). Longer time scales may also be best if

430 the goal is to catalog the entire suite of possible interspecific interactions across a range of biotic

431 and abiotic conditions (e.g., Polis, 1991).

432 Concluding Remarks. Networks that depict the accumulation of interactions across an entire

433 season or year contain the most total information about the system, but also obscure information

434 on variation that may be important to consider. We uncovered considerable temporal variability

435 in network structure that is linked to flexible species interactions within these networks - all of

436 which would be hidden with a focus only on cumulative networks. We view the results presented

437 here, and our discussion about them, to represent only one step toward resolving empirical and

438 conceptual questions about the information gained from different network analyses, the

439 appropriate temporal resolution for their study, and the deeper meanings of temporal variation

440 and flexibility in network structure. The successful exploration of these questions will rely on a

441 diversity of perspectives brought by a diversity of future researchers. If an overarching goal of

442 ecology is to understand the factors that govern the distribution of species and their abundances,

443 then a central motivation is just this: what use and what temporal resolution of networks are best

444 for improving our predictive understanding of the structure and dynamics of ecological 
communities?

447 Author's contributions. Both authors developed the project, collected the data, and wrote the

448 manuscript. The first author analyzed data, and both authors interpreted results and gave final

449 approval for publication.

450 Data availability statement. Upon acceptance of this manuscript, primary data used in analyses

451 will be archived in an appropriate public repository.

452 Declarations. The authors declare no conflict of interest.

References

455 Alarcón, R., Waser, N. M., \& Ollerton, J. (2008). Year-to-year variation in the topology of a plant456 pollinator interaction network. Oikos, 117, 1796-1807.

457 Almeida-Neto, M., Guimarães, P., Guimarães, P. R., Loyola, R. D., \& Ulrich, W. (2008). A consistent 458 metric for nestedness analysis in ecological systems: reconciling concept and measurement. Oikos, $459 \quad 117,1227-1239$.

460 Bascompte, J., \& Jordano, P. (2014). Mutualistic networks. Princeton, NJ,: Princeton University $461 \quad$ Press.

462 Bascompte, J., \& Stouffer, D. B. (2009). The assembly and disassembly of ecological networks. 463 Philosophical Transactions of the Royal Society B: Biological Sciences, 364, 1781-1787.

464 Bascompte, J., Jordano, P., Melián, C. J., \& Olesen, J. M. (2003). The nested assembly of plant465 animal mutualistic networks. Proceedings of the National Academy of Sciences USA, 100, 93834669387.

467 Bascompte, J., Jordano, P., and Olesen, J. M. (2006). Asymmetric coevolutionary networks facilitate 468 biodiversity maintenance. Science, 312, 431-433. 

$1018-1020$.

472 Blonder, B., Wey, T. W., Dornhaus, A., James, R., \& Sih, A. (2012). Temporal dynamics and network analysis. Methods in Ecology and Evolution, 3, 958-972.

474 Blüthgen, N., Menzel, F., \& Blüthgen, N. (2006). Measuring specialization in species interaction 475 networks. BMC Ecology, 6, 9-12.

476 Brosi, B., \& Briggs, H. (2013). Single pollinator species losses reduce floral fidelity and plant reproductive function. Proceedings of the National Academy of Sciences USA, 110, 13044-13048.

478 Burgos, E., Ceva, H., Perazzo, R. P. J., Devoto, M., Medan, D., Zimmermann, M., \& María Delbue, A. (2007). Why nestedness in mutualistic networks? Journal of theoretical Biology, 249, 307-313.

Burkle, L. B., \& Alarcón, R. (2011). The future of plant-pollinator diversity: Understanding 481 interaction networks across space, time, and global change. American Journal of Botany, 98, 111.

Burkle, L. B., Marlin, J. C., \& Knight, T. M. (2013). Plant-pollinator interactions over 120 years: Loss 484 of species, co-occurrence, and function. Science, 339, 1611-1615.

485 CaraDonna, P. J., Iler, A. M., \& Inouye, D. W. (2014). Shifts in flowering phenology reshape a 486 subalpine plant community. Proceedings of the National Academy of Sciences USA, 111, 49164921.

488 CaraDonna, P. J., \& Bain, J. A.. (2016). Frost sensitivity of leaves and flowers of subalpine plants is 489 related to tissue type and phenology. Journal of Ecology, 104, 55-64.

490 CaraDonna, P. J., Petry, W. K., Brennan, R. M. Cunningham, J. L., Bronstein, J. L., Waser, N. M., \& 491 Sanders, N. J. (2017). Interaction rewiring and the rapid turnover of plant-pollinator networks.

$492 \quad$ Ecology Letters, 20, 385-394.

493 Carnicer, J., Jordano P., \& Melián, C. J. (2009) The temporal dynamics of resource use by 
frugivorous birds: a network approach. Ecology, 90, 1958-1970.

Cenci, S., Song, C., \& Saavedra, S. (2018). Rethinking the importance of the structure of ecological networks under an environment-dependent framework. Ecology and Evolution, 8, 6852-6859.

497 Chittka, L., \& Thomson, J. D.. (2001). Cognitive ecology of pollination: Animal behavior and floral 498 evolution. Cambridge: Cambridge University Press.

499 Cuartas-Hernández, S., \& Medel, R. (2015). Topology of plant-flower-visitor networks in a tropical 500 mountain forest: insights on the role of altitudinal and temporal variation. PLoS One 10: e0141804.

501 Dormann, C. F. (2011). How to be a specialist? Quantifying specialization in pollination networks.

502 Network Biology, 1, 1-20.

503 Dormann, C. F., Gruber, B., \& Fründ, J. (2008). Introducing the bipartite package: Analysing

504 ecological networks. R News, 8, 8-11.

505 Dormann, C. F., Fründ, J., Blüthgen, N., \& Gruber, B. (2009). Indices, graphs and null models:

506 Analyzing bipartite ecological networks. The Open Ecology Journal, 2, 7-24.

507 Elton, C. (1927). Animal ecology. New York, NY: October House.

508 Iler, A. M., A. Compagnoni, D. W. Inouye, J. L. Williams, P. J. CaraDonna, A. Anderson, \& T. E. X. 509 Miller. (2019). Reproductive losses due to climate change-induced earlier flowering are not the 510 primary threat to plant population viability in a perennial herb. Journal of Ecology, 279, 3843-13.

511 Iler, A. M., T. T. Hoye, D. W. Inouye, \& N. M. Schmidt. (2013). Long-term trends mask variation in 512 the direction and magnitude of short-term phenological shifts. American Journal of Botany, 100, $513 \quad 1398-1406$.

514 Inouye, D. (2008). Effects of climate change on phenology, frost damage, and floral abundance on 515 montane wildflowers. Ecology, 89, 353-362.

516 Jordano, P. (1987). Patterns of mutualistic interactions in pollination and deed dispersal: connectance, 517 dependence asymmetries, and coevolution. The American Naturalist, 129, 657-677.

518 McMeans, B. C., McCann, K. S. Humphries, M., Rooney, N., \& Fisk, A. T. (2015). Food web 

variably to seasonally fluctuating environments. Ecology, 100, e02570-10.

Memmott, J., Waser, N. M., \& Price, M. V. (2004). Tolerance of pollination networks to species

524 extinctions. Proceedings of the Royal Society of London. Series B: Biological Sciences, 271, 26055252611.

526 Olesen, J. M., Bascompte, J., Elberling, H., \& Jordano, P. (2008). Temporal dynamics in a pollination network. Ecology, 89, 1573-1582. network structure and implications for estimates of specialization. Ecology Letters, 11, 564-575.

531 Pleasants, J. (1981). Bumble bee responses to variation in nectar availability. Ecology, 62, 1648-1661.

532 Poisot, T., Stouffer, D. B., \& Gravel, D. (2015). Beyond species: Why ecological interaction networks 533 vary through space and time. Oikos, 124, 243-251.

534 Polis, G. A. (1991). Complex trophic interactions in deserts: An empirical critique of food-web 535 theory. The American Naturalist, 138, 123-155.

536 R Core Team. (2018). R: A language and environment for statistical computing. Vienna: R

537 Foundation for Statistical Computing. https://www.R-project.org/.

538 Rohr, R. P., Saavedra, S., \& Bascompte, J. (2014). On the structural stability of mutualistic systems.

539 Science, 345, 1253497-1253497.

540 Saavedra, S., Stouffer, D. B., Uzzi, B., \& Bascompte, J. (2011). Strong contributors to network

541 persistence are the most vulnerable to extinction. Nature, 478, 233-235.

542 Saavedra, S., Rohr, R. P., Olesen, J. M., \& Bascompte, J. (2016a). Nested species interactions 543 promote feasibility over stability during the assembly of a polliantor community. Ecology and 
544 Evolution, 6, 997-1007.

545 Saavedra, S., Rohr, R. P., Fortuna, M. A., Selva, N., \& Bascompte, J. (2016b). Seasonal species

546 interactions minimize the impact of species turnover on the likelihood of community persistence.

547 Ecology, 97, 865-873.

548 Saavedra, S., Cenci, S., del-Val, E., Boege, K., \& Rohr, R. P. (2017). Reorganization of interaction

549 networks modulates the persistence of species in late successional stages. Journal of Animal

$550 \quad$ Ecology, 86, 1136-1146.

551 Sajjad, A., Saeed, S., Ali, M., Khan, F. Z. A., Kwon, Y. J., \& Devoto, M. (2017). Effect of temporal

552 data aggregation on the perceived structure of a quantitative plant-floral visitor network.

553 Entomological Research, 47, 380-387.

554 Schleuning, M., Fründ, J., \& García, D. (2015). Predicting ecosystem functions from biodiversity and

555 mutualistic networks: An extension of trait-based concepts to plant-animal interactions. Ecography,

$556 \quad 38,380-392$

557 Simmons, B. I., Hoeppke, C., \& Sutherland, W. J. (2019). Beware of greedy algorithms. Journal of 558 Animal Ecology, 88, 804-807.

559 Song, C., Rohr, R. P., \& Saavedra, S. (2017). Why are some plant-pollinator networks more nested 560 than others? Journal of Animal Ecology, 86, 1417-1424.

561 Song, C., Rohr, R. P., \& Saavedra, S. (2019). Beware z-scores. Journal of Animal Ecology, 88, 808562809.

563 Staniczenko, P.A., Kopp, J. C., \& Allesina, S. (2013). The ghost of nestedness in ecological networks. $564 \quad$ Nature Communications, 4, 1391.

565 Stigler, S. M. (2016). The seven pillars of statistical wisdom. Cambridge: Harvard University Press.

566 Thébault, E., \& Fontaine, C. (2010). Stability of ecological communities and the architecture of 567 mutualistic and trophic networks. Science, 329, 853-856.

568 Trøjelsgaard, K., \& Olesen, J. M. (2016). Ecological networks in motion: Micro- and macroscopic 
569 variability across scales. Functional Ecology, 30, 126-1935.

570 Valdovinos, F. S. (2019). Mutualistic networks: Moving closer to a predictive theory. Ecology

$571 \quad$ Letters, 22, 1517-1534.

572 Vázquez, D. P., Melian, C. J., Williams, N. M., Blüthgen, N., Krasnov, B. R., \& Poulin, R. (2007).

573 Species abundance and asymmetric interaction strength in ecological networks. Oikos, 116, 1120-

$574 \quad 1127$.

575 Vázquez, D. P., Blüthgen, N., Cagnolo, L., \& Chacoff, N. P. (2009). Uniting pattern and process in

576 plant-animal mutualistic networks: A review. Annals of Botany, 103, 1445-1457.

577 Vizentin-Bugoni, J., Maruyama, P. K., \& Sazima, M. (2014). Processes entangling interactions in

578 communities: Forbidden links are more important than abundance in a hummingbird-plant network.

579 Proceedings of the Royal Society B: Biological Sciences, 281, 20132397-20132397.

580 Waser, N. M., L. Chittka, L., Price, M. V., Williams, N. M., \& Ollerton, J. (1996). Generalization in

581 pollination systems, and why it matters. Ecology, 77, 1043-1060.

582 Waser, N. M., CaraDonna, P. J., \& Price, M. V. (2018). Atypical flowers can be as profitable as

583 typical hummingbird flowers. The American Naturalist, 192, 644-653.

584 Winemiller, K. O. (1990). Spatial and temporal variation in tropical fish trophic networks. Ecological 585 Monographs, 60, 331-367. 
587 Table 1. Summary of the structural properties of cumulative, season-long networks and weekly

588 networks. Cumulative values represent network metrics calculated from single point estimates

589 from cumulative, season-long networks in each year of study. Weekly values represent the mean

590 and variation in network metrics calculated from each of the weekly networks in each year.

\begin{tabular}{|c|c|c|c|c|c|c|}
\hline \multirow[t]{2}{*}{ Network parameter } & \multirow[t]{2}{*}{ Year } & \multirow[t]{2}{*}{$\begin{array}{l}\text { Cumulative } \\
\text { value }\end{array}$} & \multirow[t]{2}{*}{$\begin{array}{c}\text { Mean of } \\
\text { weekly values }\end{array}$} & \multirow[t]{2}{*}{$\begin{array}{l}\text { Range of weekly } \\
\text { values }\end{array}$} & \multicolumn{2}{|c|}{$\begin{array}{l}\text { Cumulative } \\
\text { vs. weekly } \\
\text { comparison }\end{array}$} \\
\hline & & & & & $t$ & $P$ \\
\hline \multirow[t]{3}{*}{ Connectance } & 2013 & 0.131 & 0.279 & $0.16-0.47$ & 5.35 & $<0.001$ \\
\hline & 2014 & 0.132 & 0.255 & $0.15-0.51$ & 4.49 & $<0.001$ \\
\hline & 2015 & 0.156 & 0.246 & $0.16-0.52$ & 3.17 & 0.006 \\
\hline \multirow{3}{*}{$\begin{array}{l}\text { Connectance residuals } \\
\text { (network size-adjusted) }\end{array}$} & 2013 & 0.049 & -0.004 & $-0.10-0.18$ & -2.15 & 0.055 \\
\hline & 2014 & 0.059 & -0.004 & $-0.11-0.23$ & -2.56 & 0.023 \\
\hline & 2015 & 0.122 & -0.008 & $-0.10-0.21$ & -6.17 & $<0.0001$ \\
\hline \multirow[t]{3}{*}{ Nestedness $(N O D F)$} & 2013 & 30.8 & 43.4 & $20.9-68.8$ & 2.45 & 0.032 \\
\hline & 2014 & 41.1 & 41.4 & $15.6-63.7$ & 0.08 & 0.941 \\
\hline & 2015 & 42.9 & 47.6 & $34.1-65.0$ & 1.85 & 0.086 \\
\hline \multirow{3}{*}{$\begin{array}{l}\text { Combined nestedness statistic } \\
\text { (size and connectance adjusted NODF) }\end{array}$} & 2013 & 1.75 & 2.39 & $1.51-3.51$ & 3.58 & 0.007 \\
\hline & 2014 & 2.16 & 2.47 & $1.86-3.77$ & 2.18 & 0.050 \\
\hline & 2015 & 1.75 & 2.47 & $1.84-3.36$ & 5.93 & $<0.0001$ \\
\hline \multirow[t]{3}{*}{ Network specialization $\left(\mathrm{H}_{2}{ }^{\prime}\right)$} & 2013 & 0.464 & 0.446 & $0.13-0.80$ & -0.27 & 0.793 \\
\hline & 2014 & 0.327 & 0.495 & $0.14-0.91$ & 2.49 & 0.026 \\
\hline & 2015 & 0.471 & 0.501 & $0.36-0.75$ & 0.98 & 0.356 \\
\hline \multirow[t]{3}{*}{ Robustness to plant extinction } & 2013 & 0.745 & 0.634 & $0.59-0.72$ & -8.85 & $<0.0001$ \\
\hline & 2014 & 0.751 & 0.631 & $0.53-0.72$ & -9.82 & $<0.0001$ \\
\hline & 2015 & 0.785 & 0.653 & $0.60-0.70$ & -15.62 & $<0.0001$ \\
\hline \multirow[t]{3}{*}{ Robustness to pollinator extinction } & 2013 & 0.751 & 0.703 & $0.61-0.81$ & -3.15 & 0.009 \\
\hline & 2014 & 0.767 & 0.725 & $0.59-0.86$ & -2.25 & 0.041 \\
\hline & 2015 & 0.832 & 0.739 & $0.64-0.82$ & -8.24 & $<0.0001$ \\
\hline \multirow{3}{*}{$\begin{array}{l}\text { Robustness to plant }+ \text { pollinator } \\
\text { extinction }\end{array}$} & 2013 & 0.481 & 0.372 & $0.30-0.44$ & -9.70 & $<0.0001$ \\
\hline & 2014 & 0.480 & 0.379 & $0.27-0.42$ & -8.97 & $<0.0001$ \\
\hline & 2015 & 0.517 & 0.382 & $0.25-0.43$ & -10.90 & $<0.0001$ \\
\hline
\end{tabular}



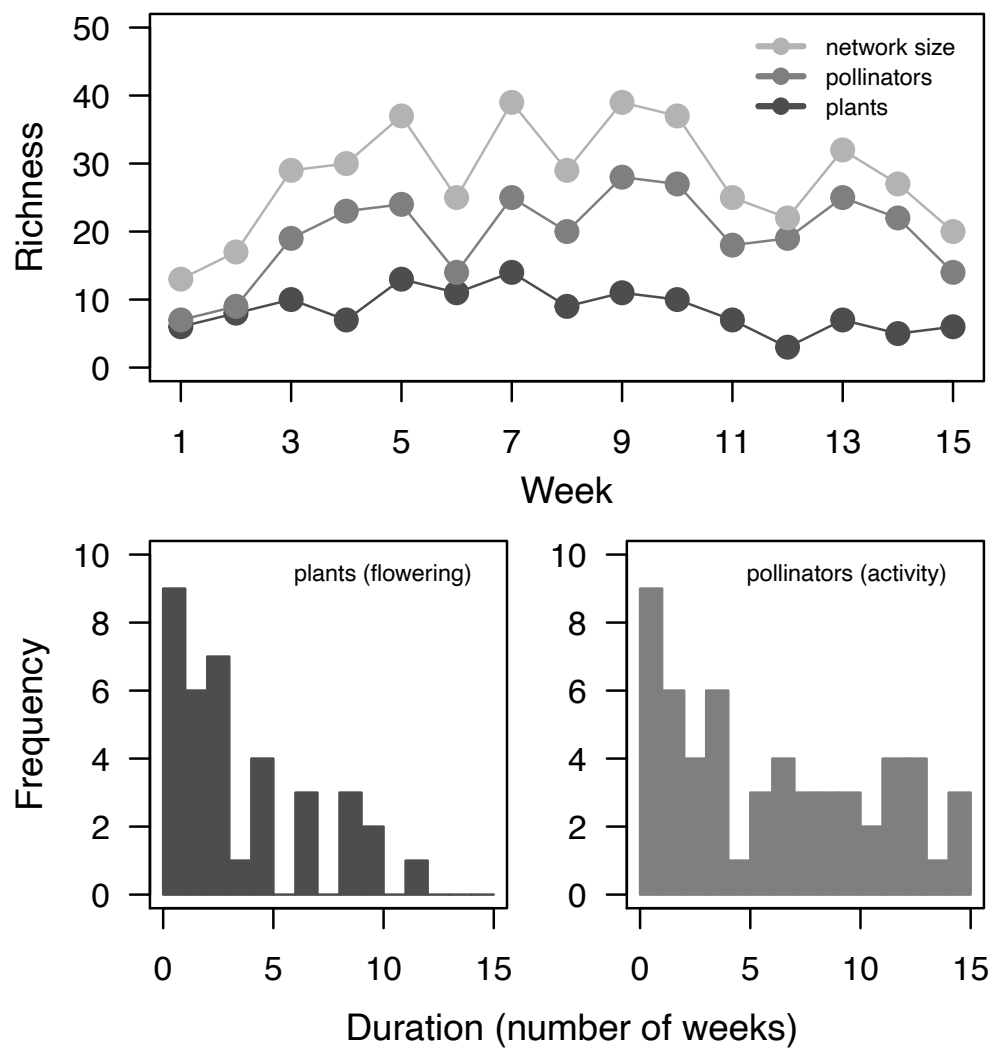

598 Figure 1. Temporal variation in species richness of plants and pollinators (top panel), and the

599 flowering duration of plants (lower left panel) and the activity duration of pollinators (lower right

600 panel), in the 2014 network (see Appendix S1: Table S2 for information additional details on plant,

601 pollinator, and interaction richness for all three summers). 


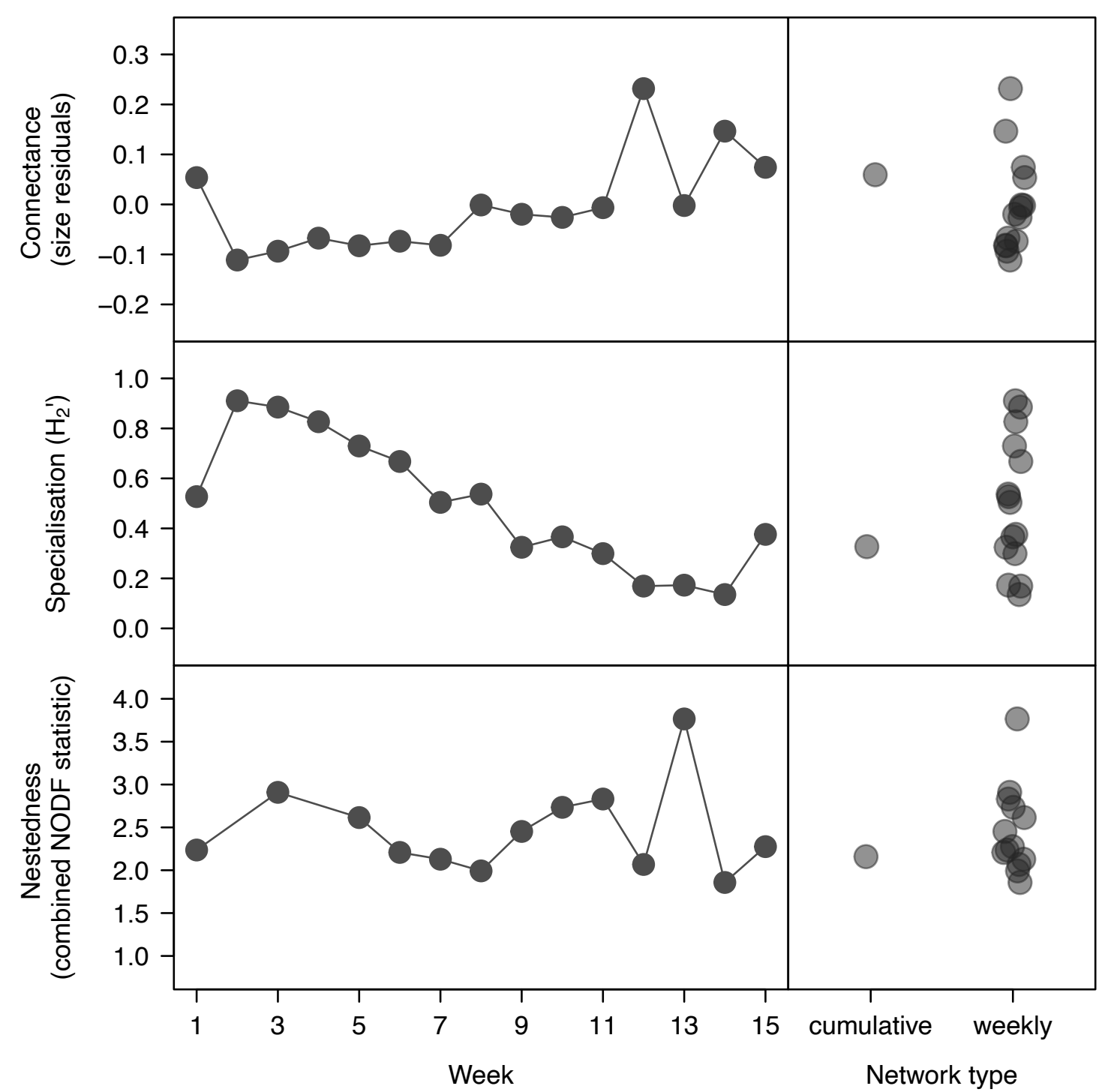

602

603 Figure 2. Temporal variation in the structure of plant-pollinator networks from week to week across

604 the 2014 growing season (left panels) and comparison of cumulative, season-long network metrics

605 with weekly metrics (right panels). Data for the other two summers are shown in Figs. S2, S3.

606 Missing values for the combined nestedness statistic represent cases where calculations were not

607 possible because there were fewer links in the network than the number of species.

608 


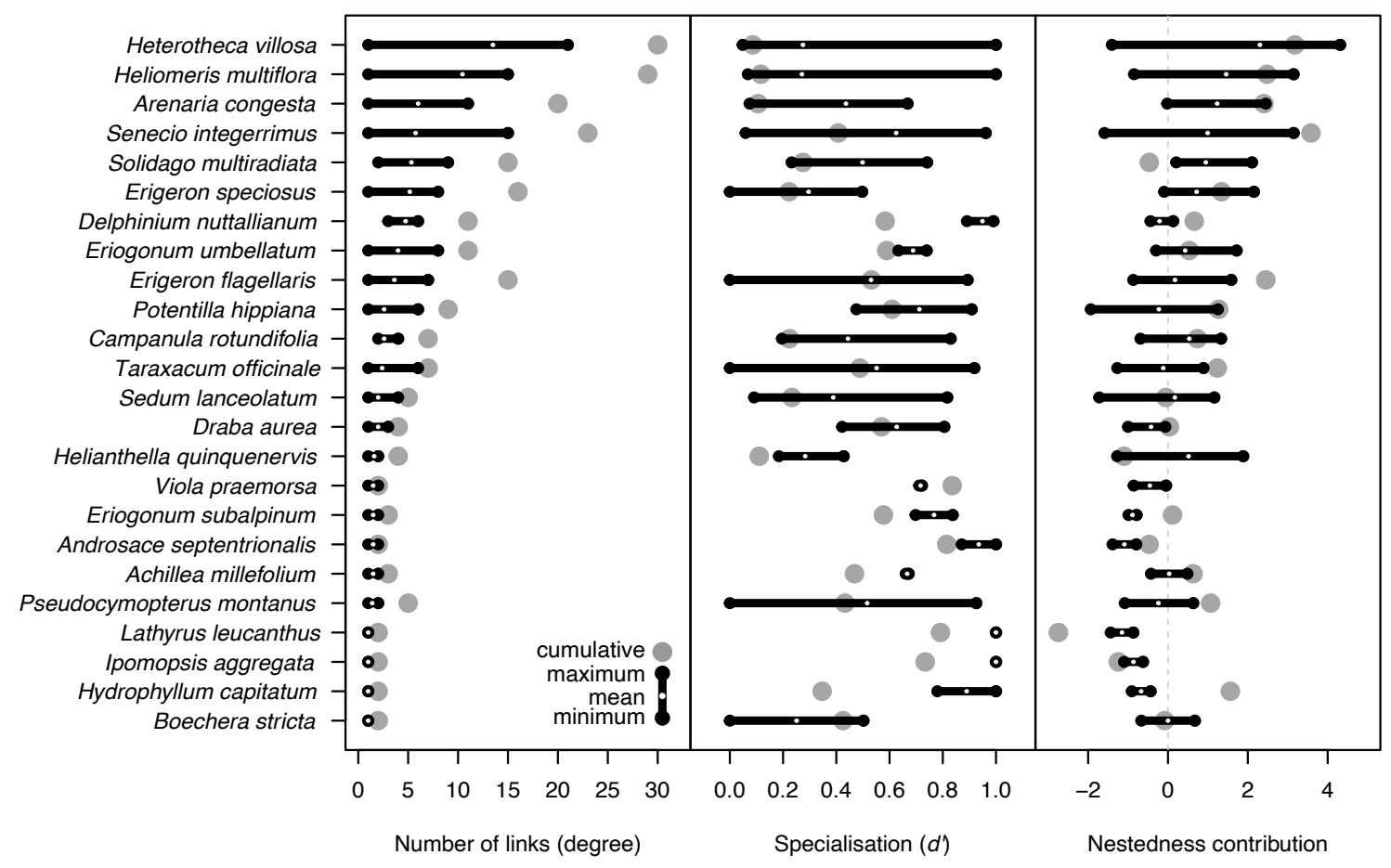

609

610 Figure 3. Temporal variation in plant species network roles: the number of links per plant species

611 (left panel), interaction specialization (middle panel), and contribution to nestedness (right panel).

612 Data are from the 2014 growing season; the other two summers are shown in Figs. S3, S4. Black dots

613 and lines represent the range of weekly values; small white dots represent the mean of weekly values;

614 and large grey dots represent cumulative values. Nestedness contribution values are z-scores

615 generated from each network; if values overlap with zero this indicates that a species' contribution

616 ranges from positive to negative; it is not to be interpreted as having an overall neutral effect. 


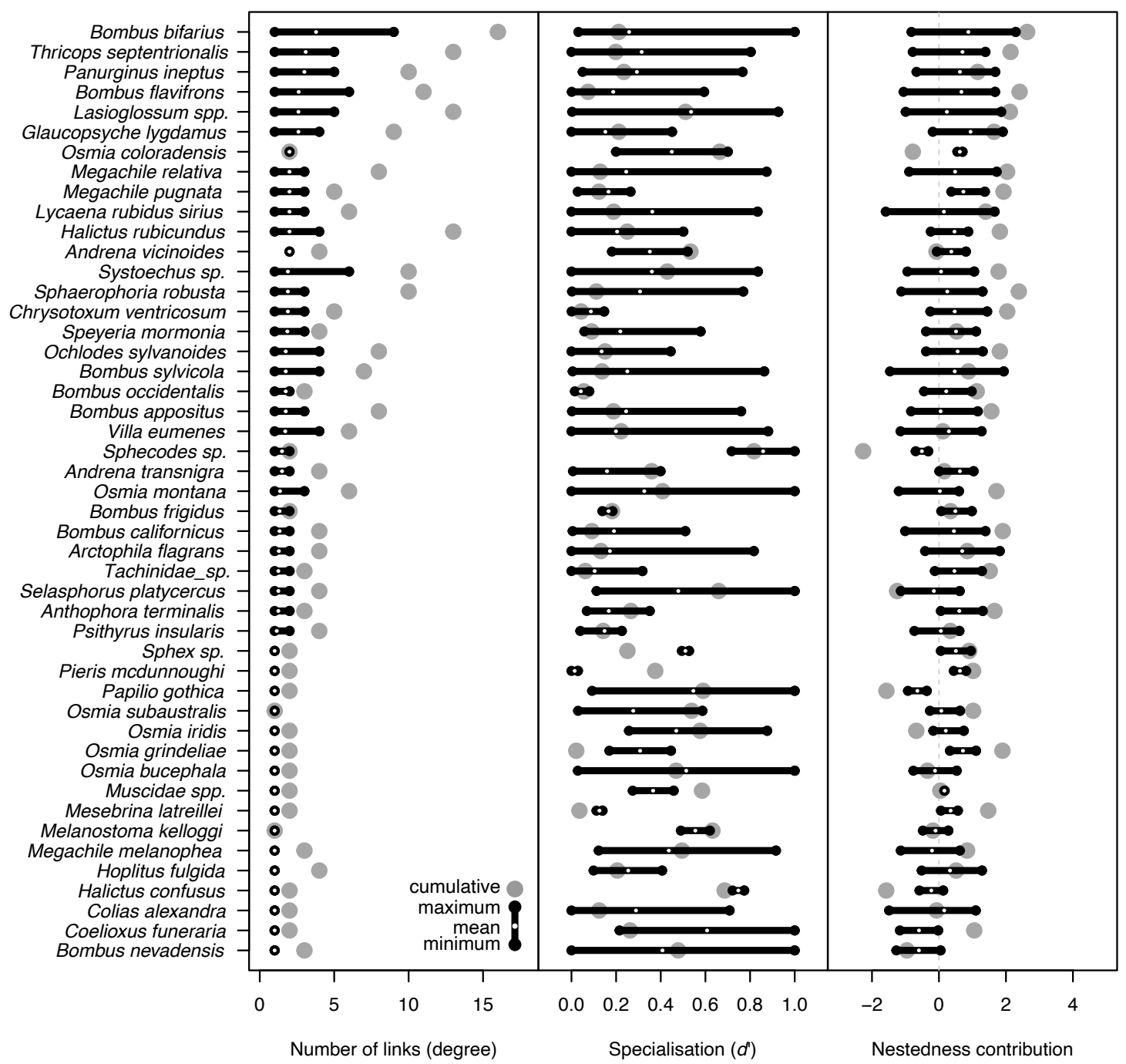

618

619 Figure 4. Temporal variation in pollinator species interaction roles within networks: the number of

620 links per pollinator species (left panel), interaction specialization (middle panel), and contribution to

621 nestedness (right panel). Data are from the 2014 growing season; the other two seasons are shown in

622 Figs. S5 and S6. Other conventions follow Figure 3. 


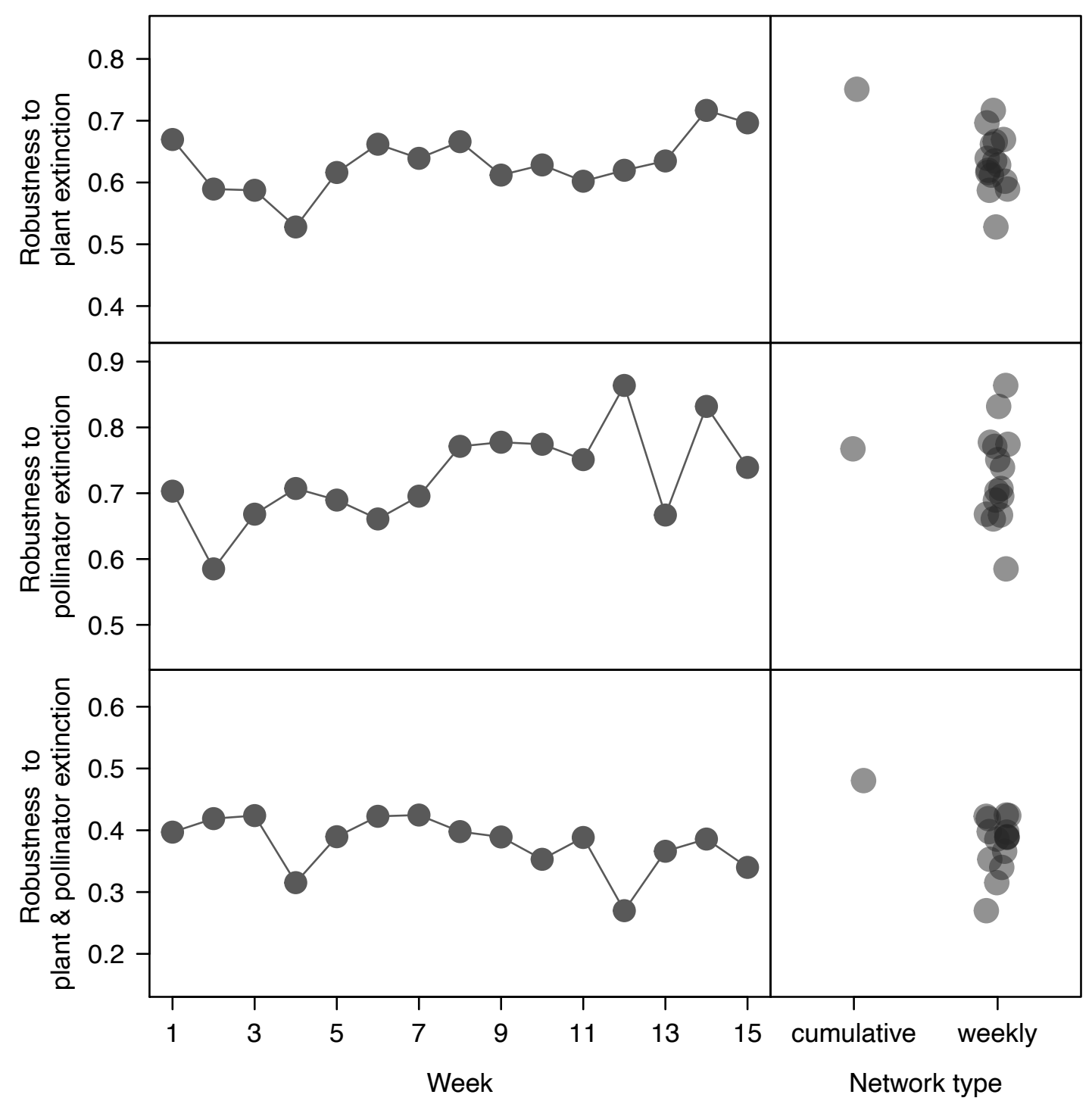

623

624 Figure 5. Potential network robustness to simulated random extinction of plants, pollinators, and

625 plants and pollinators for the 2014 growing season. Potential robustness values represent the area

626 underneath the extinction simulation curve where 0 indicates a network that collapses via secondary

627 extinction after the first primary extinction and 1 indicates a network that is completely robust to

628 primary extinctions (i.e., no secondary extinctions even after all primary extinctions). Data for the

629 other two seasons are shown in Figs. S8 and S9. 